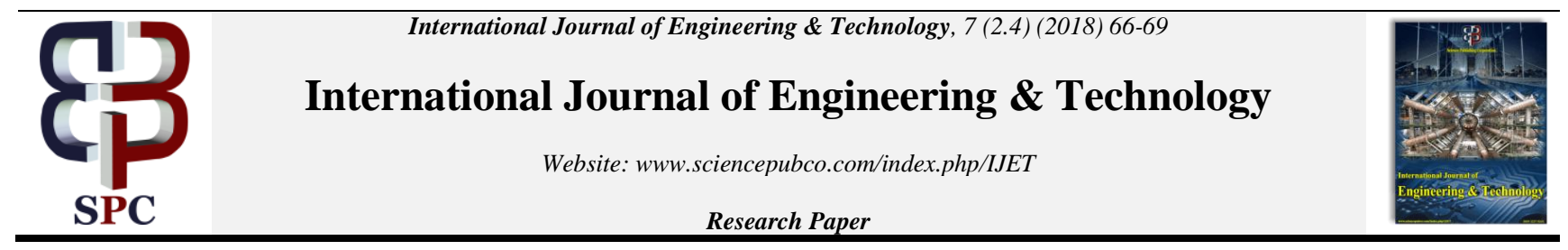

\title{
Successive Duplicate Detection in Scalable Datasets in Cloud Database
}

\author{
N. Rajkumar ${ }^{1}$, K. Kishore Kumar ${ }^{2}$, J. Vivek ${ }^{3}$ \\ ${ }^{1}$ Associate Professor, ${ }^{2,3}$ Assistant Professor, Department of Computer Science and Engineering, School of Computing \\ Vel Tech Rangarajan Dr. Sagunthala R\&D Institute of Science and Technology, Avadi, Chennai-62, TamilNadu \\ *Corresponding author E-mail:sivarajkumar@gmail.com
}

\begin{abstract}
Replica identification is the path toward perceiving various depictions of same true matters. Today, duplication location methodologies needed process ever greater datasets in ever shorter time: keeping this idea on dataset ends up being dynamically troublesome. To present, dynamic duplicate and distinguishing by proof figuring happened to be using Progressive Sorted Neighbourhood Method and Progressive Blocking augmentations more profitability occurred in order to find duplicates. If the execution time is restricted then the grow of general technique considers the time accessible and generates reports that considerably produces results faster than ordinary systems. Broad examinations exhibit that our dynamic counts can twofold the capability after some season of standard copy recognition and basically improve related work.
\end{abstract}

Keywords: Duplication Detection, Dataset, Progressive Blocking, Progressive Sorted Neighbourhood Method, Data cleaning

\section{Introduction}

Everything considered, information mining is the way toward dissecting information from trade points of view and compacting it into pleasing data - data that can be utilized to build pay, cuts costs, or both. Data mining composing PC programs is one of various lucid instruments for isolating information. It enables clients to isolate information from a broad assortment of estimations or centres, mastermind it, and pack the affiliations saw. To be sure, information mining is the way toward discovering affiliations or cases among various fields in broad social databases.

Hile huge scale data headway has been making separate exchange and exact structures; information mining gives the relationship between the two. Information mining programming isolates affiliations and cases in set away exchange information in context of open-finished client request. A couple of sorts of symptomatic writing computer programs are available: authentic, machine learning, and neural frameworks.

Classes: Put away information is utilized to find information in foreordained gatherings. For instance, an eatery network could mine client buy information to decide when clients visit and what they regularly arrange. This data could be utilized to build movement by having day by day specials.
Clusters: Information things are gathered by sensible connections or buyer inclinations. For instance, information can be pitted to distinguish advertise sections or buyer attractions.

Associations: Information can be mined to recognize affiliations. The brew diaper illustration is a case of cooperative mining.

Sequential patterns: Information is mined to envision conduct examples and patterns. For instance, an open air hardware retailer could anticipate the probability of a rucksack being bought in light of a buyer's buy of resting packs and climbing shoes.

Different levels of analysis are available: Simulated neural systems: Non-direct prescient models that learn through preparing and look like natural neural systems in structure.

Genetic algorithms: Improvement procedures that utilization procedure, for example, hereditary mix, changes, and common choice in a plan in light of the ideas of characteristic development.

Nearest neighbour method: A system that orders each record in a dataset in view of a mix of the classes of the $\mathrm{k}$ record(s) most like it in a verifiable dataset (where $\mathrm{k}=1$ ). Now and again called the $\mathrm{k}$ closest neighbour strategy.

Rule induction: The abstraction of helpful if then guidelines from information in view of measurable criticalness.

Data visualization: Designs devices are utilized to show information connections. This task utilizes the idea of information 
mining. Its Aim is to recognize the copy sections officially present or made by client in datasets to make the information, mistake free and ever usable in the event of recovery of information from the database.

Information are among the most critical resources of an organization. However, because of information changes and messy information passage, mistakes, for example, copy sections may happen, making information purging and specifically copy recognition basic. Client has little information about the given information. Taking above issue, I will roll out an extra improvement in the program to chip away at adaptable datasets which will build the exactness and rightness of the program for distinguishing copy sections and lessening the likelihood of making false reports, in this manner helping in enhanced disclosure of learning from information.

Much research on copy discovery, otherwise called substance determination and by numerous different names concentrates on combine choice calculations that endeavor to expand review from one viewpoint and proficiency then again. The most unmistakable estimations around their approaches to planned finest $\mathrm{k}$ closeness connect that accepts a remarkable document construction for evaluating connection hopefuls. This advance consistently settles replicas and moreover encourages the limitation issue. Pay As You Go entity decision and displayed three sorts of dynamic replica area methodologies, called "intimations". A client has just constrained, perhaps obscure time for information purging and needs to make most ideal utilization of it. At that point, essentially begin the calculation and end it when required. The outcome size will be boosted. A client has little learning about the given information yet at the same time needs to design the purging procedure. A client needs to do the cleaning intuitively to, for example, discover great arranging keys by experimentation. At that point, run the dynamic calculation over and over; each run rapidly reports perhaps extensive outcomes. All exhibited clues create static requests for the examinations and miss the chance to powerfully modify the correlation arrange at runtime in light of transitional outcomes.

\section{Proposed Methodology}

In this work, be that as it may, we concentrate on dynamic calculations, which endeavor to report most matches at an opportune time, while conceivably marginally expanding their general runtime. To accomplish this, they have to gauge the similitude of all examination hopefuls with a specific end goal to look at most encouraging record matches first. We suggest, dynamic copy zone checks that holds PSN Method, that provides finest on clean datasets and nothing and Progressive Blocking, performs good on gigantic as well as astoundingly unsanitary datasets. Together improves the ability of copy zone on gigantic datasets. We suggest the above mentioned approaches, which uncover different attributes and outflank current approaches. We show a concurrent dynamic approach for the multi-pass framework and change an incremental transitive conclusion calculation that together structures the essential finish dynamic copy disclosure work process. It dynamic approach for the multi-pass system and adjust an incremental transitive conclusion tally that together structures the fundamental finish dynamic copy ID work process. We depict a novel quality measure for dynamic copy affirmation to fairly rank the execution of various rationalities. We totally study on two or three honest to goodness datasets testing our own particular and past figuring.
This examination overhauled early quality and same possible quality. Our tallies approaches consistently alter their lead through thusly picking immaculate parameters, e.g., window sizes, square sizes, and coordinating keys, rendering their manual particular senseless. Thusly, we out and out encourage the parameterization unpredictability for duplicate area when all is said in done and add to the headway of more customer instinctive applications.

\section{System Architecture}

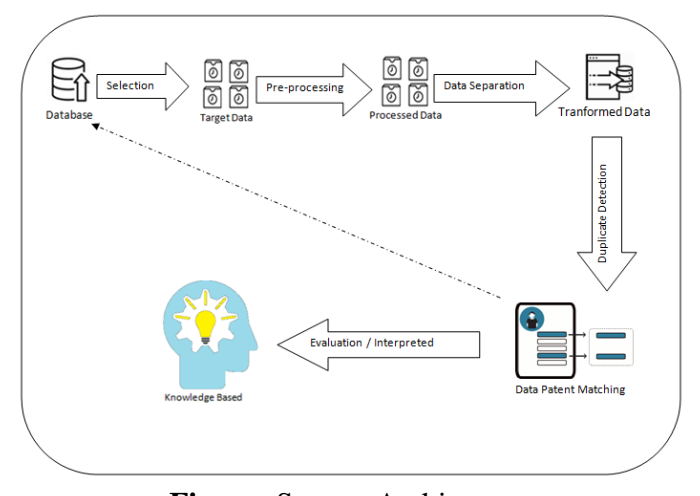

Figure: System Architecture

The DFD is additionally called as air pocket graph. It is a straightforward graphical formalism that can be utilized to speak to a framework regarding input information to the framework, different handling completed on this information, and the yield information is produced by this framework. The information stream graph (DFD) is a champion among the most fundamental showing gadgets. It is used to show the system parts. These fragments are the system technique, the data used by the methodology, an external substance that interfaces with the structure and the information streams in the structure. DFD demonstrates how the information goes through the structure and how it is changed by a movement of changes. It is a graphical strategy that portrays information stream and the progressions that are associated as data moves from commitment to yield. DFD is generally called bubble diagram. A DFD may be used to address a structure at any level of pondering. DFD may be partitioned into levels that address growing information stream and useful detail.

\section{Related Works}

Cloud foundations draw in the fruitful parallel execution of information certified assignments, for example, substance affirmation on wide datasets. We explore difficulties and conceivable plans of utilizing the MapReduce programming model for parallel part affirmation. Specifically, we propose and study two MapReduce-based use for Sorted Neighborhood disquieting that either utilize distinctive MapReduce occupations or apply a remarkably fitted information replication.

Tuple link is the course toward sorting out tuples from two or three databases that propose similar substances. Precisely when related on a solitary database, this system is known as reduplication. Constantly, arranged information are persuading the chance to be essential in different application zones, since they can contain data that isn't accessible something other than what's expected, or that is unreasonably finished the best, making it difficult to get. Evacuating copy records in a solitary database is a fundamental 
advance in the information cleaning process, since copies can incredibly influence the outcomes of any ensuing information arranging or information mining. With the developing size of the present databases, the multifaceted idea of the arranging strategy ends up one of the important inconveniences for record linkage and de-duplication [3]. Starting late, extraordinary requesting systems have been made for record linkage and reduplication. They are away to decrease the amount of record sets to be taken a gander at in the planning methodology by emptying clear no matching sets, while meanwhile keeping up high organizing quality. This paper exhibits an examination of 12 varieties of 6 asking for structures. Their adaptable quality is bankrupt down, and their execution and adaptability is assessed inside a trial structure utilizing both composed and true blue edifying gatherings. No such point by point outline has so far been dispersed.

Duplication detection is the course to find various tuples to a dataset that address a similar certifiable material. By virtue of the gigantic expenses of a broad examination, ordinary figuring select just encouraging record sets for association. Two procedures are windowing and blocking. Blocking techniques appropriate into windowing frameworks, disjoint subsets especially the Sorted Neighborhood Method, slide a window over the engineered tuples and inspect tuples essentially inside the window. We demonstrate another estimation called Sorted Blocks in several assortments, which sums up both methodologies [10]. To overview Sorted Blocks, we have driven far reaching examinations with various datasets. These demonstrate that our new calculation needs less associations with locate a similar number of copies.

The closeness of copy records is a basic information quality worry in clearing databases. To perceive copies, substance confirmation for the most part called duplication divulgence or record linkage is utilized as a touch of the information cleaning framework to see records that possibly propose a similar certifiable segment. We exhibit the Stringer framework that gives an assessment structure to understanding what pieces stay towards the objective of to a great degree versatile and widely profitable duplication ID calculations [7]. In this paper, we utilize Stringer to study the possibility of the packs (social affairs of potential copies) got from a few unconstrained assembling figuring utilized as a bit of show with unforgiving join frameworks. Our work is incited by the present fundamental developments that have made wrong join tallies essentially adaptable. Our wide assessment uncovers that some social occasion estimations that have never been considered for copy territory, perform massively well like both precision and flexibility.

The issue of uniting different databases of information about customary components is a great part for time experienced in KDD and decision helping applications in tremendous business and government affiliations. The issue we think about is regularly called the Merge/Purge issue and difficult to understand both in scale and exactness. Immense stores of data regularly have different duplicate information sections about comparative components that are difficult to isolate together without a sharp "equational speculation" that recognizes relative things by a brain boggling, space subordinate planning process[5]. We have developed a structure for accomplishing this Data Cleansing errand and show its use for purging courses of action of names of potential customers in a prompt publicizing compose application. Our results for verifiably created data are had all the earmarks of being exact and effective when taking care of the data different conditions using various keys for organizing on every dynamic pass. Brushing results of individual overlooks using transitive conclusion the self-sufficient results, conveys essentially more exact results at cut down cost. The structure gives a choose programming module that is definitely not hard to program and extremely awesome at finding duplicates especially in an area with tremendous measures of data. This paper inconspicuous components upgrades in our structure, and reports on the productive use for a certifiable database that convincingly favors our results heretofore proficient for quantifiably delivered data.

\section{Implementation and Result Discussion}

\section{Dataset Collection}

To gather and additionally recover information about exercises, results, setting and different variables. It is essential to think the sort of data it need to assemble from the members and the ways to examine that data. The informational collection relates to the substance of a solitary database table, or a solitary factual information lattice, where each segment of the table speaks to a specific variable. Subsequent to gathering the information to store the Database.

\section{Pre-processing Method}

Information Pre preparing or Data maintenance, Data is rinsed throughout procedures, for example, fulfilling in misplaced esteems, levelling the loud information, or settling the irregularities in information. And furthermore utilized to expelling the undesirable information. Usually utilized as a preparatory information mining practice, information pre-processing changes the information into a configuration that will be all the more effortlessly and adequately handled with the client end goal.

\section{Data Separation}

Ensuing to finishing the pre-handling, data division is to carried out. The shutting figurings dole out each record to a settled assembling of related records (pieces) and a short time later dissect all arrangements of records inside these social events. Each piece inside the square connection network addresses the examinations of all tuples in a solitary square with all tuples in another tuple, the 17 central impeding; every pieces contain a comparative size.

\section{Duplicate Detection}

The copy identification regulations set by the overseer, the framework cautions the client probable copies when the client attempting to make fresh records or refresh previous records. To keep up information originality, you be able to plan a copy discovery employment to check for copies for all records that match specific criteria. You be able to clean the information by erasing, disabled, or blending the copies detailed by a copy location.

\section{Quality Measures}

The nature of these frameworks is, henceforth, estimated utilizing money saving advantage count. Particularly for customary copy identification forms, it is hard to meet a spending restriction, in light of the fact that their runtime is difficult to anticipate. By conveying whatever number copies as could be expected under the circumstances in a given measure of time, dynamic procedures improve the money saving advantage proportion. In assembling, a measure of magnificence or a condition of being free from imperfections, inadequacies and huge varieties. It is realized by strict what's more, steady sense of duty regarding certain principles that accomplish consistency of an item with a specific end goal to fulfill particular client or client prerequisites. 


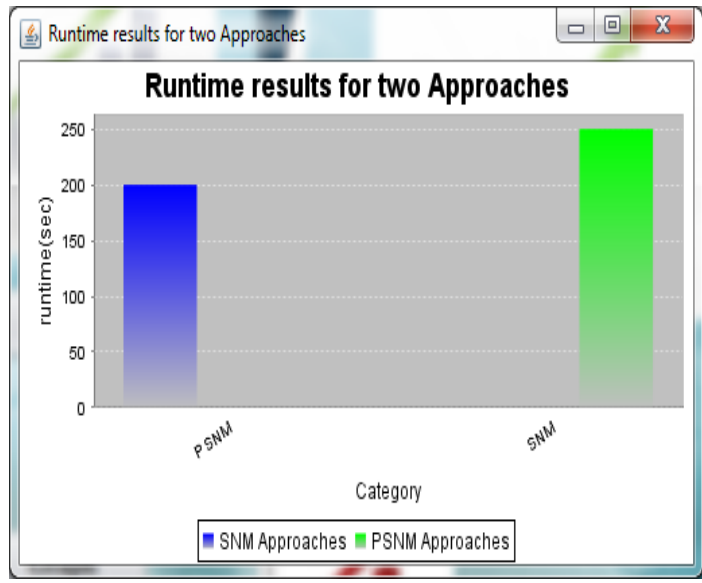

Figure: Runtime results for two Approaches

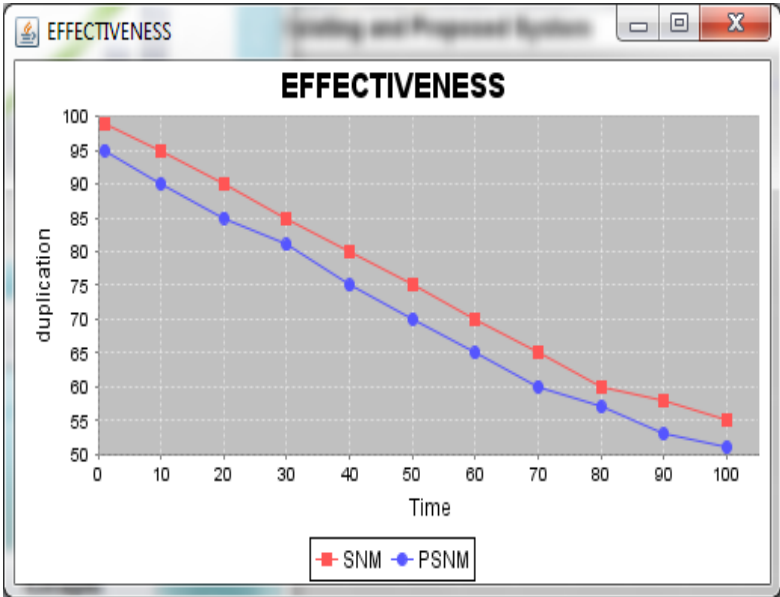

Figure: Comparison of Existing and Proposed System

\section{Conclusion}

This paper presented the dynamic arranged neighbourhood technique and dynamic blocking. The two calculations increment the effectiveness of copy identification for circumstances with restricted execution time; they powerfully change the positioning of examination applicants in view of middle of the road results to execute promising correlations first and less encouraging examinations later. To pick the execution get of our calculations, we present a novel quality measure for progressiveness on headings to be perfect in existing measures. Utilizing this measure, tests showed that our approachs beat the consistent SNM by up to 100 percent and related work by up to 30 percent for the change of an absolutely novel copy zone work process, we proposed a dynamic engineering technique, Magpie, a dynamic multi-pass execution show up, Attribute Concurrency, and an incremental transitive conclusion estimation. The changes AC-PSNM and ACPB utilize diverse sort keys at the same time to interleave their dynamic cycles. By investigating halfway outcomes, the two methods consistently rank the specific sort keys at runtime, surely reassuring the key affirmation issue. In future work, we need to join our dynamic methodology with adaptable frameworks for copy affirmation to pass on happens fundamentally speedier. Specifically, Kolb et al. shown a two stage parallel SNM, which executes a conventional SNM on adjusted, covering parts. Here, we can rather utilize our PSNM to reliably discover copies in parallel.

\section{References}

[1] T.Senthil Murugan, Jagannath E Nalavade, "C-mixture and multiconstraints based genetic algorithm for collaborative data publishing," Elsevier - Journal of King Saud University Computer and Information Sciences (Article in press), 2016

[2] Kavitha R, Rajkumar N, Kannan E."Framework for Primary Health Centers (PHC)" using cloud in the International Journal named Discovery, using Cloud. Discovery, , 30(116), 17-21 2015.

[3] P. Christen, "A survey of indexing techniques for scalable record linkage and deduplication," IEEE Trans. Knowl. Data Eng., vol. 24, no. 9, pp. 1537-1555, Sep. 2012.

[4] R. Kavitha, E. Kannan and S. Kotteswaran "Implementation of Cloud based Electronic Health Record (EHR) for Indian Healthcare Needs" in the International journal of Science and Technology., Vol 9(3), DOI: 10.17485/ijst/2016/v9i3/86391, January 2016

[5] M. A. Hernandez and S. J. Stolfo, "Real-world data is dirty: Data cleansing and the merge/purge problem," Data Mining Knowl. Discovery, vol. 2, no. 1, pp. 9-37, 1998.

[6] T.Senthil Murugan, Jagannath E Nalavade, "THRFuzzy: Tangential holoentropy-enabled rough fuzzy classifier to classification of evolving data streams", Springer - Journal of Central South University,Vol.24(8), 2017

[7] O. Hassanzadeh, F. Chiang, H. C. Lee, and R. J. Miller, "Framework for evaluating clustering algorithms in duplicate detection," Proc. Very Large Databases Endowment, vol. 2, pp. 1282-1293, 2009.

[8] Kavitha R, E Kannan "A Novel Triangular Boundary based classification approach to detect outliers and predict the class labels using the kernel Methods" by Journal of Information Science and Engineering.

[9] T.Senthil Murugan, Jagannath E Nalavade, "HRNeuro-fuzzy: Adapting neuro-fuzzy classifier for recurring concept drift of evolving data streams using rough set theory and holoentropy", Journal of King Saud University - Computer and Information Sciences, (Article in press), 2016

[10] U. Draisbach and F. Naumann, "A generalization of blocking and windowing algorithms for duplicate detection," in Proc. Int. Conf. Data Knowl. Eng., 2011, pp. 18-24. 